\title{
THE STRESS BEHAVIOUR OF POWERED VEHICLE BODY
}

\author{
G.VIJAY PRAKASH, A.V.RATNA PRASAD
}

\begin{abstract}
The stream around body (powered vehicle, transports, trucks) under typical working condition is basically fierce. It is regularly portrayed by substantial scale division and distribution areas, a mind boggling wake stream, long trailing vortices and cooperation of limit layer stream on vehicle and ground. In growing new street vehicle basic for planner for see completely its assembly of stream around to the vehicle. It will have impact on such important element as the state of vehicle, streamlined drag, fuel utilization, clamor generation and street dealing with. Generally, vehicle planner have picked up their comprehension of the wind current around a vehicle through broad breeze burrow testing. Body-on-outline is a vehicle development strategy a different body is fixed on a generally inflexible casing or body conveying in the motor \& drivetrain. The first strategy for structure cars, body-on-outline development is currently utilized for the most part for fueled vehicle. Car streamlined features includes the investigation of optimal design of street vehicles. Its fundamental objectives are diminishing drag, limiting clamor outflow, improving efficiency, averting undesired lift powers and limiting different reasons for streamlined insecurity at high speeds. In the current project cfd analysis of powered vehicle's outer body is done at 30kmph. Design of frame done using NX-CAD software and CFD analysis runs through Ansys CFD package.
\end{abstract}

\section{INTRODUCTION}

\subsection{BODY FRAME}

The center component of any vehicle is the body structure. The vehicle body interfaces all the diverse segments; it houses the drive train and above all conveys and ensures travelers and payload. The body structure should be inflexible to help weight and stress and to safely integrate every one of the parts. Besides, it must oppose and mellow the effect of a collide with securely ensure the inhabitants. Also, it should be as light as conceivable to enhance efficiency and execution.

Favorable circumstances

Simpler to configuration, construct and alter (less of an issue since PC helped structure (CAD) is typical, yet at the same time leverage for mentor assembled vehicles).

Calmer, in light of the fact that the anxieties don't go into the body, which is disconnected from the casing with elastic cushions around the connection jolts. This is less huge now, yet generally bodies would squeak and shake more as they

Revised Manuscript Received on July 05, 2019.

G.VIJAY PRAKASH Acharyanagarjuna universcity, Nagarjuna Nagar-522510

DR.A.V.RATNA PRASAD Principal of V.R .Siddardha Engineering College. Kannur, Vijayawada.

Email.vikram14391439@gmail.com rusted, ointments depleted, and clasp relaxed. Secluded bodies were influenced to a lesser degree by these methods of maturing.

Less demanding to fix after mishaps. This is critical for people on call (police, flame, EMS) and taxis, in light of the fact that harmed jolt on bumpers can be supplanted in the company's very own carport for frivolous money, with the taxi came back to acquiring status quickly, while a unibody body would require rectifying by paid experts on a machine costly to lease - with the taxi laid up for fix longer. Terrific Am permits rounded spaceframe vehicles to supplant their unibody partners, as the autos can without much of a stretch be fixed with new clasps.

Can enable a maker to effectively subcontract parts of work, for example as when Austin subcontracted the aluminum body work of the Austin A40 Sports to Jensen Motors.

Can permit more torsional flexing before yielding (trucks, truck-base SUVs going 4x4 romping)

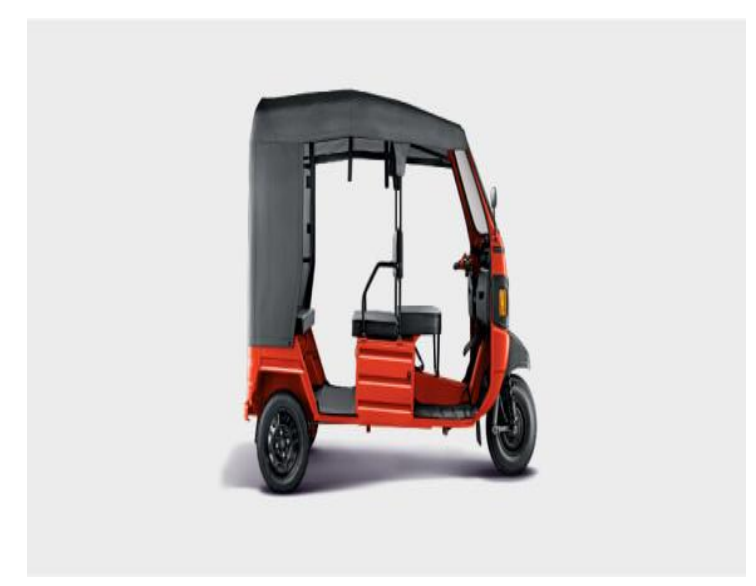

\subsection{OBJECTIVES:}

In the current project cfd analysis of powered vehicle's outer body is done at $30 \mathrm{kmph}$. Design of frame done using NX-CAD software and CFD analysis runs through Ansys CFD package.

\subsection{INTRODUCTION TO UNIGRAPHICS}

The Unigraphics NX Modeling application gives a strong demonstrating framework to empower fast reasonable structure. Specialists can consolidate their prerequisites and plan limitations by characterizing numerical

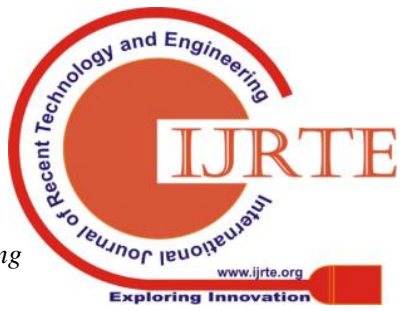


connections between various pieces of the structure.

Configuration specialists can rapidly perform theoretical and point by point structures utilizing the Modeling highlight and limitation based strong modeler. They can make and alter mind boggling, reasonable, strong models intelligently, and with far less exertion than increasingly conventional wire edge and strong based frameworks. Highlight Based strong displaying and altering capacities enable originators to change and refresh strong bodies by straightforwardly altering the elements of a strong component or potentially by utilizing other geometric altering and development systems.

It serves the fundamental structure undertakings by giving diverse work seats. The essential work seats in UNIGRAPHICS are

Part structure

Wire edge and surface plan

Get together structure

Drafting

\subsection{ANSYS CFD INTRODUCTION}

Computational liquid elements (CFD) is the exploration of foreseeing liquid stream, heat exchange, mass exchange, concoction responses, and related wonders by unraveling the scientific conditions which oversee these procedures utilizing a numerical procedure. The consequence of CFD examinations is pertinent building information utilized in: Conceptual investigations of new structures. - Detailed item advancement. - Troubleshooting. - Redesign. - CFD examination supplements testing and experimentation. Reduces the all out exertion required in the research center

\section{Utilizations of CFD}

- Flow and warmth move in modern procedures (boilers, heat exchangers, burning gear, siphons, blowers, channeling, and so forth.).

- Aerodynamics of ground vehicles, flying machine, rockets.

- Film covering, thermoforming in material handling applications.

- Flow and warmth move in drive and power age frameworks.

- Ventilation, warming, and cooling streams in structures.

- Chemical vapor statement (CVD) for incorporated circuit producing.

- Heat exchange for gadgets bundling applications.

\section{LITERATURE REVIEW}

Ahmed et al. (1984) [2] analysed time averaged wake structure around the Ahmed body at a Reynoldsnumber equal to $1.2 * 106$ by manipulating the rear slant angle in the range of 0-400in increments of 50. However, the study did not yield information about unsteady flow characteristics of the flow around the Ahmed body.

Bayraktar et al. (2001) [3] examined the external aerodynamics of Ahmed reference body for the rear slant angles of $0,12.5$, and 25 degrees. The main concern was observing the effect on the lift and drag coefficients due to variation of Reynolds number and calculating wind-averaged drag coefficients. The 10-inch water column possessing an electronic scanning module coupled with a force balance system was utilized to measure the pressure and forces which are consequently used to calculate the drag coefficients.

Spohn and Gillieron (2002) [4]carried out experiments to investigate the flow characteristics of the Ahmed body with the slant angle equal to 25 degree and the Reynolds number equal to $8.3 * 103$. The experimentwas carried out in a closed water tunnel, using the electrolyte precipitation technique at the front as well as therear of the Ahmed body.

M. Zunaid, Naushad Ahmad Ansari [5]designed and implemented the curved boat tail configuration

without the AFC device on a generic SUV design working on the principle of Coanda effect, causing the airflow over and under the SUV to take a curved path resulting in blowing of air in the immediate wake region. They examined its effect on various aerodynamic parameters and reported an increase in the average pressure in the wake region along with a significant drag reduction of $8.013 \%$.

Morelli et al. (1976) conceptualized and proposed a theoretical method to determine the optimum shape

of a passenger car body for minimum drag by imposing the condition that the total lift be zero. This study proved that the aerodynamic drag can be reduced substantially without utilizing any additional devices by optimizing the body shape. Morelli et al. (2000) [6] proposed a new technique called "fluid tail", obtainable by addressing small power air jets in the wake, for application to the aerodynamic design of basic shape of a passenger car. .

P. Drage et al (2008) [7]conducted both CFD simulations as well as wind tunnel testing (T.U Graz windtunnel) on the Ahmed body with two rear slant angle configurations. The simulation was performed with the Fluent and by using the Reynold Stress Model. The mesh was developed with the meshing tool SPIDER. It consisted of 4 prism layers around the body and had an unstructured volume mesh giving a total cell count of 7.7 million cells. This type of meshing approach is commonly adopted as the time spent to create a structured grid around real vehicle shapes would prevent engineers from operating within the strict deadlines imposed by vehicle design cycles. For the $25^{\circ}$ angle, a drag coefficient of 0.299 and a lift coefficient of 0.345 was obtained from the wind tunnel experiment, while the CFD simulation resulted in the values of 0.295 and 0.387 for drag

and lift coefficient respectively. The RSM model showed an over estimation of the pressure coefficient over the slant angle, when compared to the pressure measurement made by Lienhart and Becker (2003). [8]

\section{PROBLEM DEFINITION}

Body-on-frame is automobile construction methods of a separate body is mounted on a relatively rigid frame or chassis carrying in to the engine and drivetrain. The

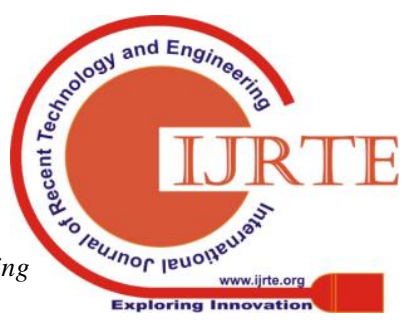


original method of building the automobiles, body-on-frame construction is used mainly for powered vehicle. Automotive aerodynamics comprises of the study of aerodynamics of road vehicles. Its main goals are reducing drag, minimizing noise emission, improving fuel economy, preventing undesired lift forces and minimizing other causes of aerodynamic instability at high speeds.

\section{3D MODELLING OF POWERED VEHICLE'S OUTER FRAME}

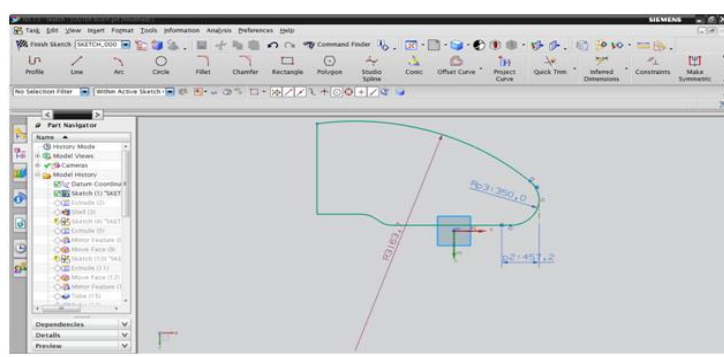

Fig.4.1: 2D sketch of vehicle's outer body frame

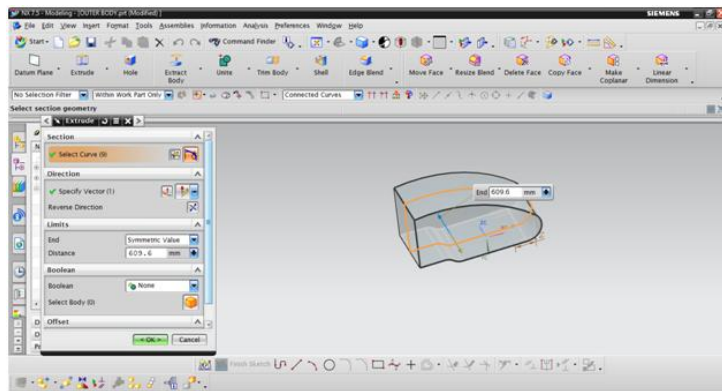

Fig.4.2 Extrude of 2D sketch of vehicle's outer body frame

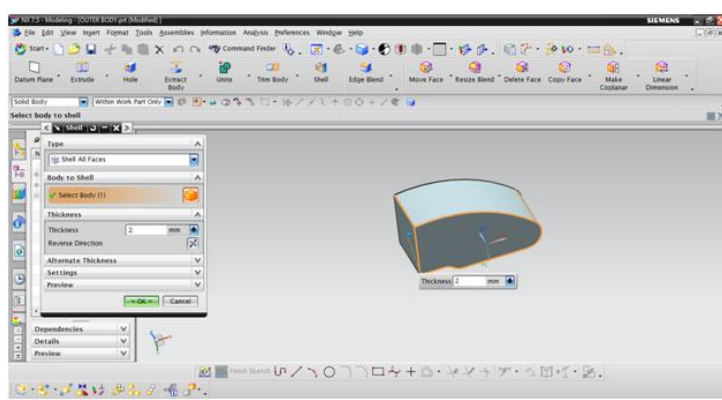

Fig.4.3 Extrude of 2D sketch of vehicle's outer body frame

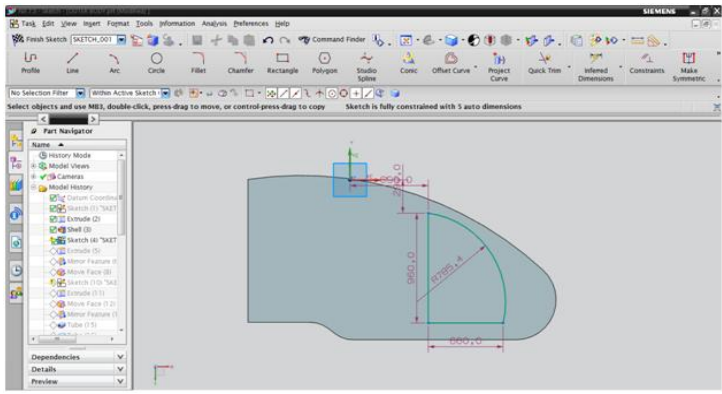

Fig.4.4: 2D sketch of vehicle's outer body frame

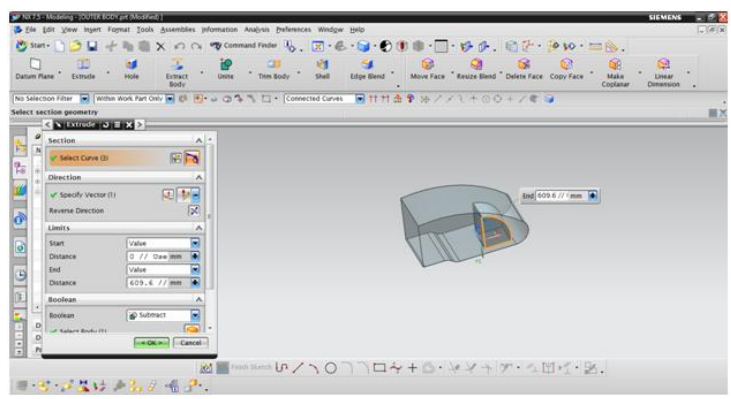

Fig.4.5: Extrude of 2D sketch of vehicle's outer body frame

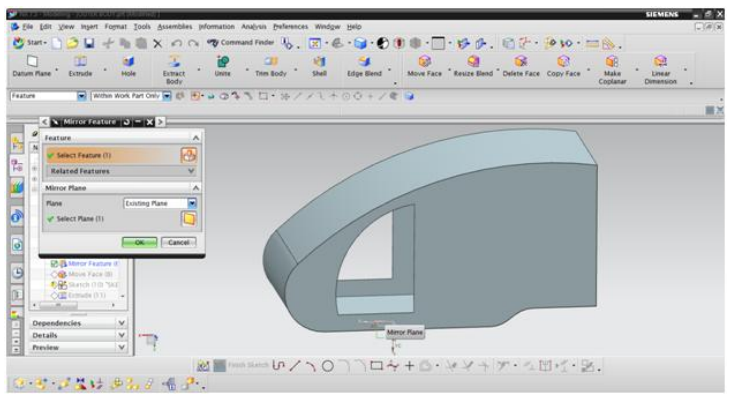

Fig.4.6: Mirror of 2D sketch of vehicle's outer body frame

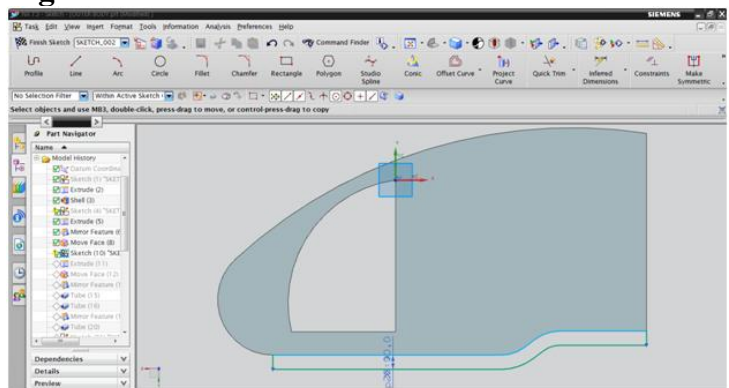

Fig.4.7: 2D sketch of vehicle's outer body frame

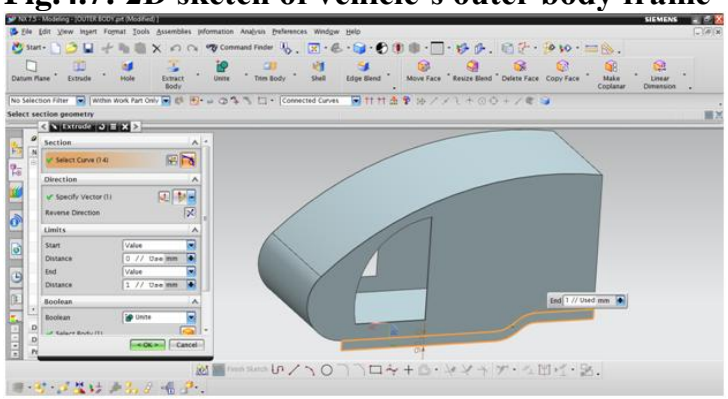

Fig.4.8: Extrude of above sketch

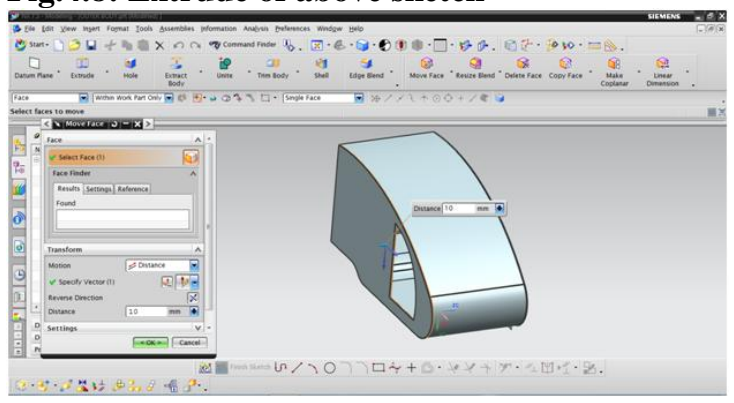

Fig.4.9: Extrude of 2D sketch of vehicle's outer body frame 


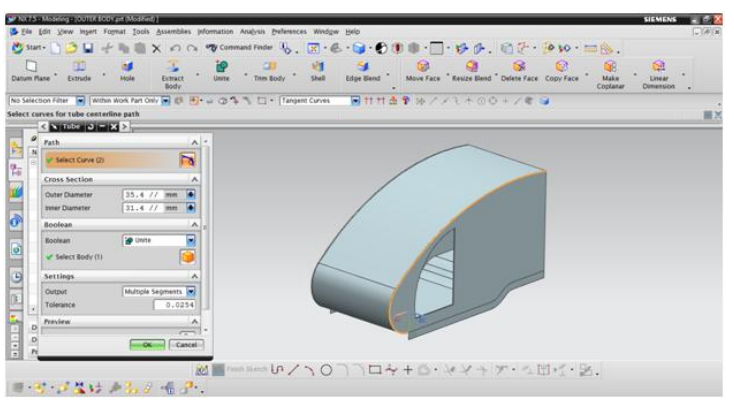

Fig.4.10: Extrude of 2D sketch of vehicle's outer body frame

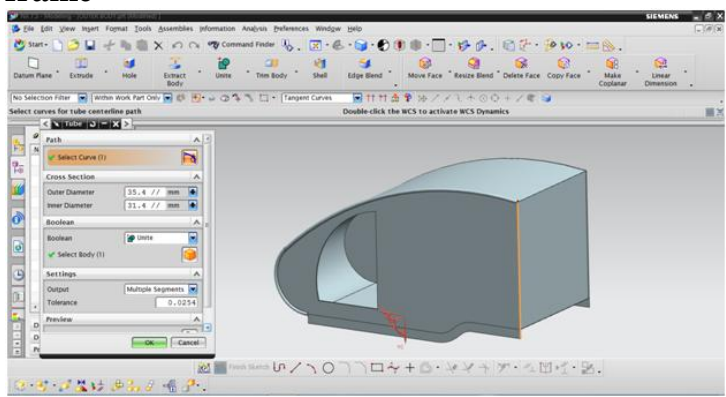

Fig.4.11: Extrude of 2D sketch of vehicle's outer body frame

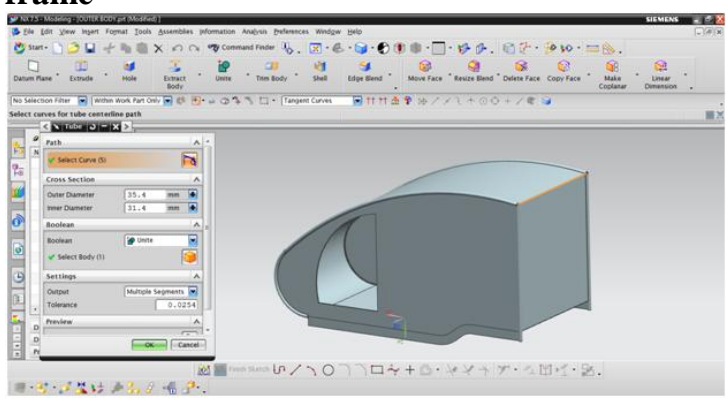

Fig.4.12: Extrude of 2D sketch of vehicle's outer body frame

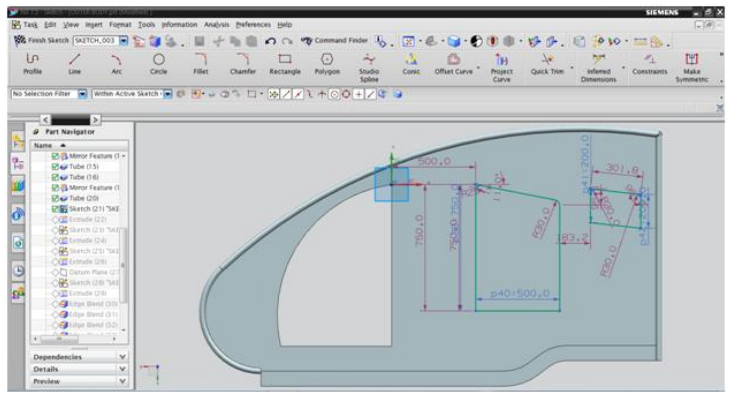

Fig.4.13: 2D sketch of vehicle's outer body frame

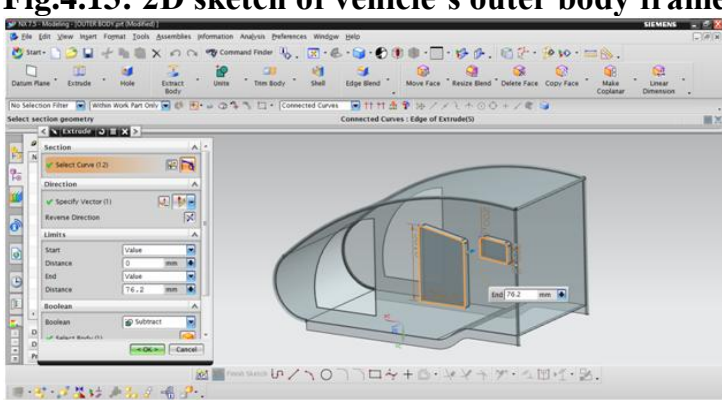

Fig.4.14: Extrude of 2D sketch of vehicle's outer body frame

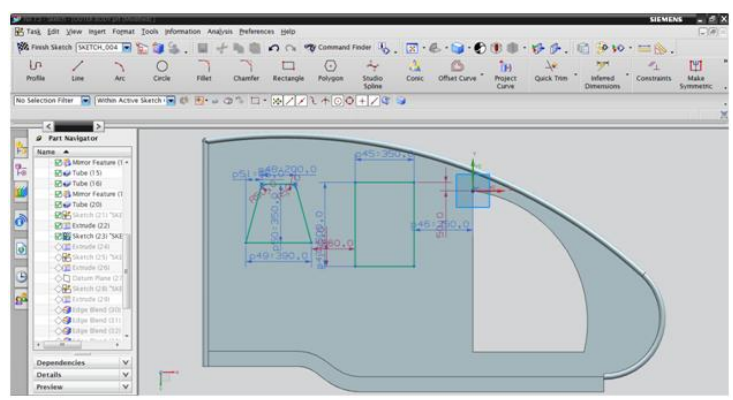

Fig.4.15: 2D sketch of vehicle's outer body

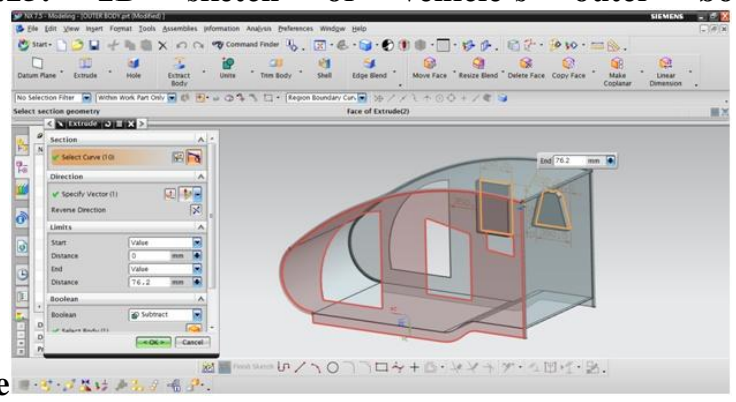

Fig.4.16: Extrude of 2D sketch of vehicle's outer body frame

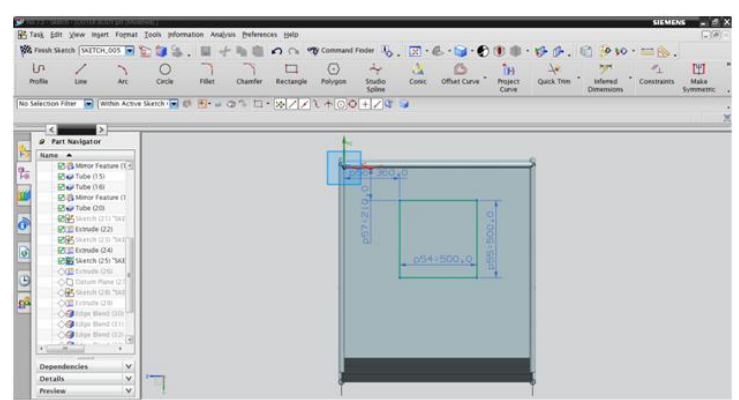

Fig.4.17: 2D sketch of vehicle's outer body frame

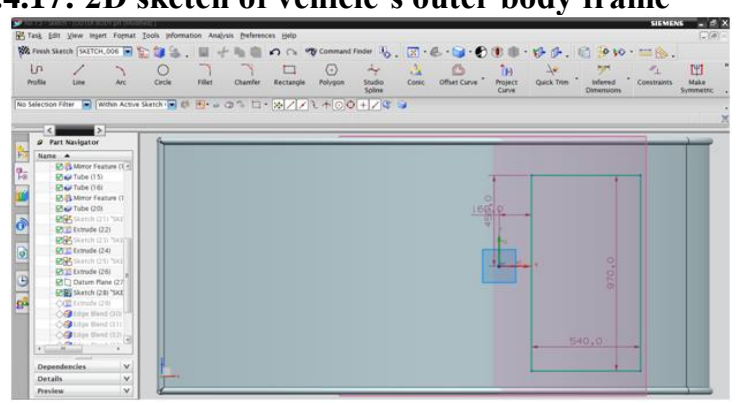

Fig..4.18 2D sketch of vehicle's outer body frame

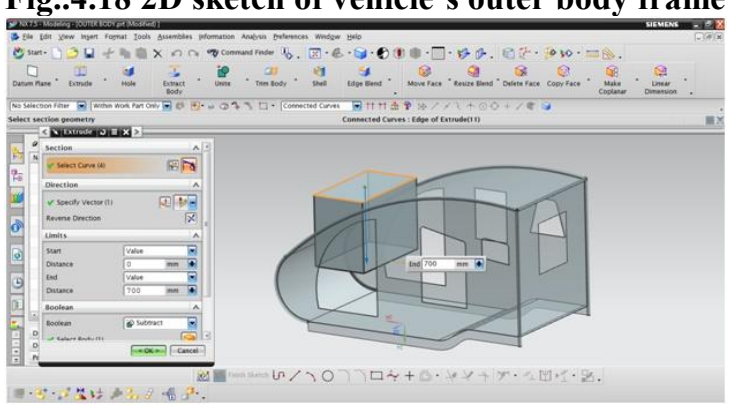

Fig..4.19 Extrude of 2D sketch of vehicle's outer body frame

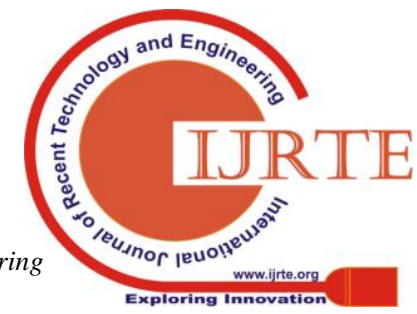




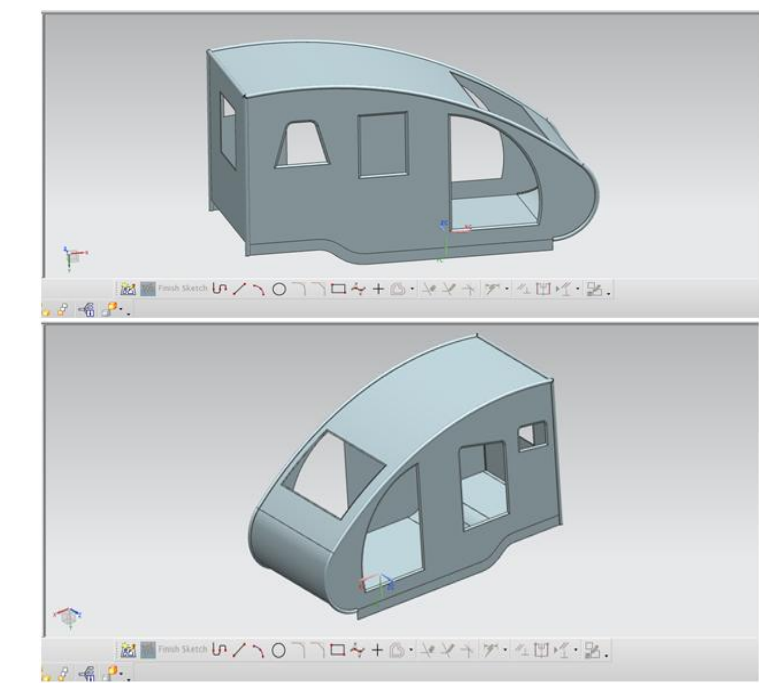

Fig..4.20 Final vehicle's outer body frame V.CFD ANALYSIS OF BODY FRAME AT 30KMPH SPEED

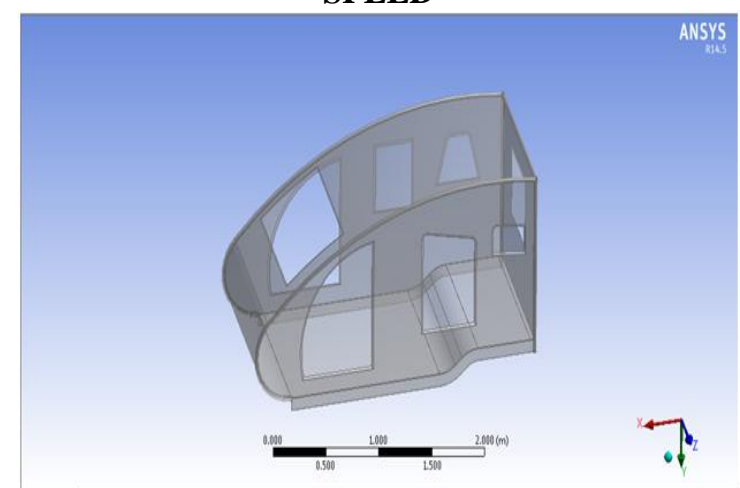

Fig.5.1 Imported designed body frame in AnsysCFD

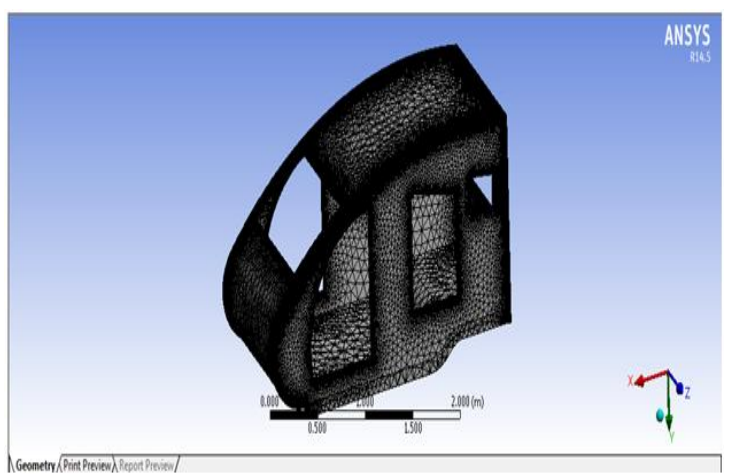

Fig.5.2 Meshed model

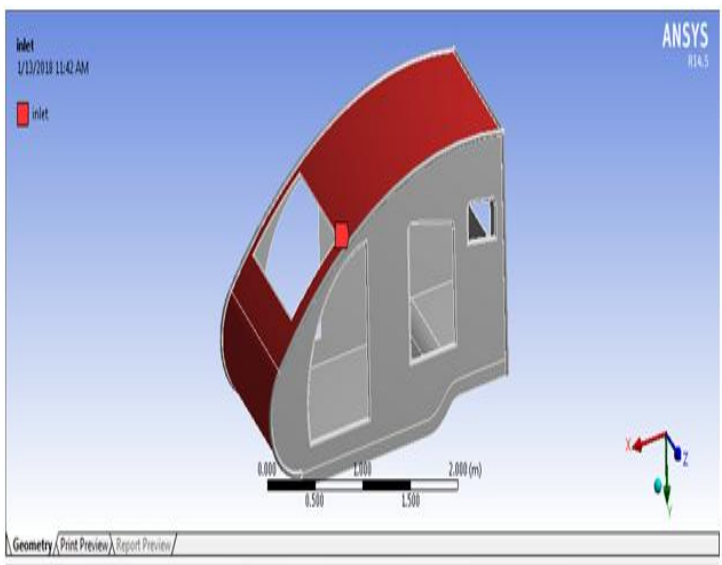

Fig. 5.3 Applied inlet condition

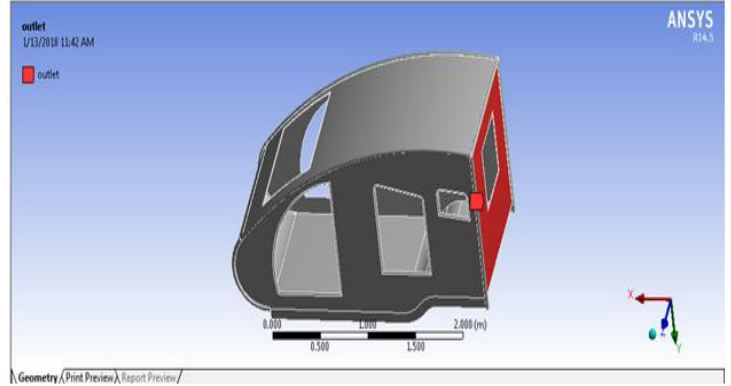

Fig .5.4 Applied outlet condition

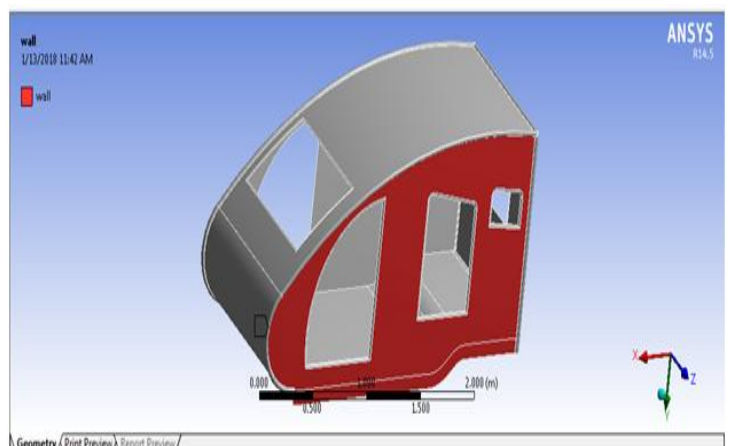

Fig.5.5 Applied wall condition

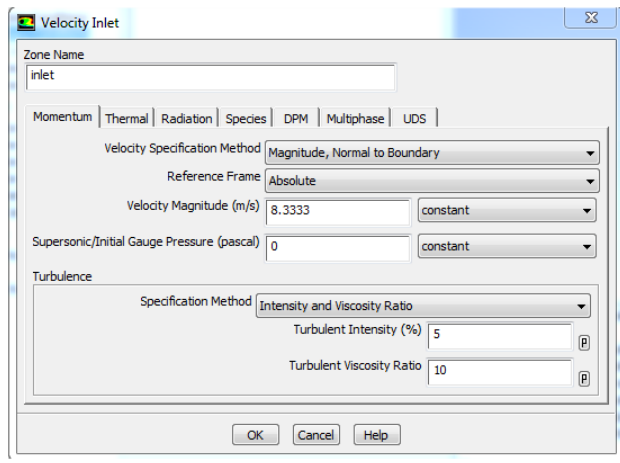

Fig.5.6 Applied inlet velocity condition

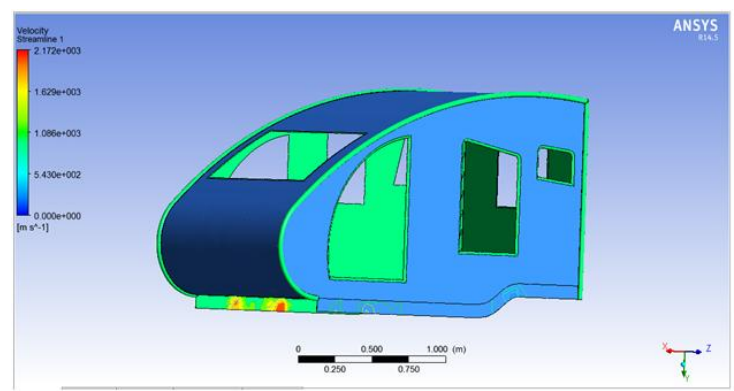

Fig.5.7Stream velocity results

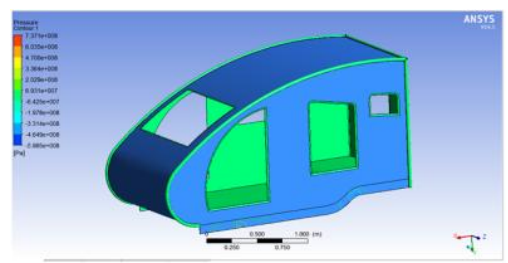

Fig.5.8 Stream pressure results

Published By:

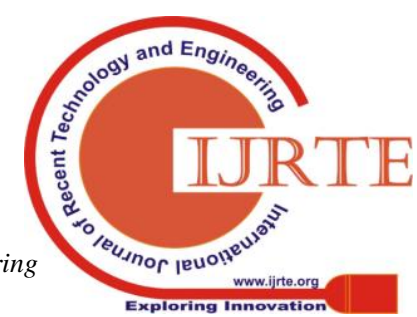




\section{CONCLUSION}

$>$ The Air velocity and the Max Avg. stream pressure observed on the outer body frame for applied loading condition is $2.17 \mathrm{e} 3 \mathrm{~m} / \mathrm{s}$ and $737 \mathrm{MPa}$ with respectively.

\section{REFERENCES}

[1]. „Sustainable ground transportation - review of technologies, challenges and opportunitiesse, Ramesh K. Agarwal, Department of Mechanical Engineering \& Materials Science, Washington University in St. Louis, MO 63130, USA.

[2]. „Some Salient Features of the Time - Averaged Ground Vehicle Wake", (SAE-TP-840300) Ahmed, S.R., Ramm, G., Faitin, G.,

1984. Society of Automotive Engineers, Inc., Warrendale, PA.

[3]. „Experimental and Computational Investigation of Ahmed Body for Ground Vehicle Aerodynamics"e, I. Bayraktar, D. Landman,

and O. Baysal, SAE Technical Paper 2001-01-2742, 2001, doi:10.4271/2001-01-2742.

[4]. ,Flow Separations Generated by a Simplified Geometry of an Automotive Vehicle"e, A.Spohn, P. Gillieron, IUTAM Symposium:

Unsteady Separated Flows, April 8-12, 2002, Toulouse, France.

[5]. „Numerical Study of the Generic Sports Utility Vehicle Design with a Drag Reduction Add-On Device",Shubham Singh, $M$.

Zunaid, Naushad Ahmad Ansari, ShikhaBahirani, SumitDhall, Sandeep Kumar, Department of Mechanical Engineering, Delhi

Technological University, Hindawi Publishing Corporation Journal of Computational Engineering Volume 2014, Article ID

\section{4.}

[6]. „A New Aerodynamic Approach to Advanced Automobile Basic Shapes", A. Morelli, SAE Technical Paper 2001-01-0491, 2001.

[7]. „Efficient Use of Computational Fluid Dynamics for the Aerodynamic Development Process in the Automotive Industry" ${ }^{\text {ee }}$ P. Drage,

A. Grabiel and G. Lindbichler, AIAA 2008-6735.

[8]. „Flow and turbulence structures in the wake of a simplified car model (Ahmed Model) ${ }^{\mathrm{ec}}$, H. Lienhart, C. Soots and S. Becker, SAE

Technical Paper 2003-01- 0656, 2003.

[9]. ,Optimization of bluff body for minimum Drag in ground proximity"e, T. Han, D. C. Hammond, C. J. Sagi, AIAA Journal, Vol. 30,

No. 4 (1992), pp. 882-889, doi: 10.2514/3.11005.

[10]. „Characterization of a square back Ahmed body near wake flow"e, A. Lahayea, A. Leroya\& A. Kourtaa, $21^{\prime}$ 'eme Congress Francis

deM'ecanique.
[11]. „Best practice guidelines for handling Automotive External Aerodynamics with FLUENT ${ }^{e e}$, Version 1.2 (Feb 9th 2005), Marco

Lanfrit, Fluent Deutschland GmbH Birkenweg 14a 64295 Darmstadt/Germany.

[12]. „Investigation of relationship between drag and lift coefficients for a generic car model ${ }^{\mathrm{ee}}$, Ivan Dobrev, FawazMassouh,

BULTRANS-2014, Sep 2014, Sozopol, Bulgaria.BULTRANS $2014 \quad$ Proceedings, pp.171-174.<Hal-01082895>

[13]. ,Experimental investigation and numerical simulation of the flow around an automotive model: Ahmed body"e, Ivan Korkischko\&

Julio Romano Meneghini, 19th International Congress of Mechanical Engineering, November 5-9, 2007, Brasília, DF. [14]. „Numerical modeling of airflow over the Ahmed body”, Y. Liu, Alfred Moser Air and Climate Group, Swiss Federal Institute of

Technology, ETH-Zentrum WET A1, CH-8092 Zurich, Switzerland.

[15]. „Fundamental of Aerodynamicse, 5th edition, John D. Anderson, McGraw-Hill Book Co 